\title{
REMARK ON THE INEQUALITY OF F. RIESZ
}

\author{
WŁODZIMIERZ EENSKI \\ Faculty of Mathematics, Informatics and Econometry, University of Zielona Góra \\ Szafrana 4a, 65-516 Zielona Góra, Poland \\ E-mail:w.lenski@wmie.uz.zgora.pl
}

\begin{abstract}
We prove F. Riesz' inequality assuming the boundedness of the norm of the first arithmetic mean of the functions $\left|\varphi_{n}\right|^{p}$ with $p \geq 2$ instead of boundedness of the functions $\varphi_{n}$ of an orthonormal system.
\end{abstract}

1. Inequality of F. Riesz. Let $\left(\varphi_{k}\right)$ be an orthonormal system in $[a, b]$, that is,

$$
\begin{gathered}
\int_{a}^{b} \varphi_{k}(t) \varphi_{n}(t) d t=0 \quad(k, n=1,2,3, \ldots), \\
\int_{a}^{b}\left|\varphi_{k}(t)\right|^{2} d t=1 \quad(k=1,2,3, \ldots),
\end{gathered}
$$

where $\varphi_{k} \in L_{[a, b]}^{2}(k=1,2,3, \ldots)$, and let

$$
a_{k}(f)=\int_{a}^{b} f(t) \varphi_{k}(t) d t \quad(k=1,2,3, \ldots)
$$

be the sequence of Fourier coefficients of a function $f \in L_{[a, b]}^{2}$ with respect to the system $\left(\varphi_{n}\right)$. The well known result of F. Riesz states

Theorem 1 (F. Riesz [4]). Let

$$
\left|\varphi_{k}(t)\right| \leq M \quad \text { for almost all } t \in[a, b] \text { and } k=1,2,3, \ldots
$$

with $M$ independent of $k$, and let $p \in(1,2]$ and $p^{\prime}$ be such that $1 / p+1 / p^{\prime}=1$.

$$
1^{\circ} \text { If } f \in L_{[a, b]}^{p} \text { then }
$$

$$
\left(\sum_{k=1}^{\infty}\left|a_{k}(f)\right|^{p^{\prime}}\right)^{1 / p^{\prime}} \leq M^{\frac{2-p}{p}}\left(\int_{a}^{b}|f(t)|^{p} d t\right)^{1 / p}
$$

2000 Mathematics Subject Classification: Primary 42C05; Secondary $30 \mathrm{~B} 62$.

The paper is in final form and no version of it will be published elsewhere. 
$\mathscr{2}^{\circ}$ If $\left(a_{k}\right) \in l^{p}$ then there exists $f \in L_{[a, b]}^{p^{\prime}}$ such that $a_{k}=a_{k}(f)$ and

$$
\left(\int_{a}^{b}|f(t)|^{p^{\prime}} d t\right)^{1 / p^{\prime}} \leq M^{\frac{2-p}{p}}\left(\sum_{k=1}^{\infty}\left|a_{k}(f)\right|^{p}\right)^{1 / p} .
$$

A generalization of this result was obtained by J. Marcinkiewicz and A. Zygmund [2], where a condition on the $L_{[a, b]}^{q}(2<q \leq \infty)$ norm of the functions $\varphi_{k}$ was used, and the constant $M$ was replaced by a sequence of constants $M_{k}$ (see also [3] p. 166).

In the present note we consider another slightly more general condition on the system $\left(\varphi_{k}\right)$. We suppose the boundedness of the first arithmetic mean of the $L_{[a, b]}^{q}(2 \leq q \leq \infty)$ norms of the functions $\varphi_{k}$ instead of their boundedness.

THEOREM 2. Let

$$
\sum_{k=1}^{n}\left(\int_{a}^{b}\left|\varphi_{k}(t)\right|^{q} d t\right)^{p^{\prime} / q} \leq M^{p^{\prime}} n(b-a)^{p^{\prime} / q} \quad \text { when } q<\infty
$$

and

$$
\sum_{k=1}^{n}\left|\varphi_{k}(t)\right|^{p^{\prime}} \leq M^{p^{\prime}} n \quad \text { for almost all } t \in[a, b] \text { when } q=\infty
$$

with $M$ independent of $n, q \geq p^{\prime}, \varphi_{k} \in L_{[a, b]}^{q}$ for every $k=1,2,3, \ldots$ with $p^{\prime} \geq 2$ and let $p \in(1,2]$ such that $1 / p+1 / p^{\prime}=1$.

$1^{\circ}$ If $f \in L_{[a, b]}^{p}$ then

$$
\left(\sum_{k=1}^{\infty}\left|a_{k}(f)\right|^{p^{\prime}}\right)^{1 / p^{\prime}} \leq M^{\frac{2-p}{p}}\left(\int_{a}^{b}|f(t)|^{p} d t\right)^{1 / p} .
$$

$2^{\circ}$ If $\left(a_{k}\right) \in l^{p}$ then there exists $f \in L_{[a, b]}^{p^{\prime}}$ such that $a_{k}=a_{k}(f)$ and

$$
\left(\int_{a}^{b}|f(t)|^{p^{\prime}} d t\right)^{1 / p^{\prime}} \leq M^{\frac{2-p}{p}}\left(\sum_{k=1}^{\infty}\left|a_{k}(f)\right|^{p}\right)^{1 / p} .
$$

2. Proof of Theorem 2. We will use the same notation as in the book [1]. The main part of the proof is the same as in [1, Theorem 6.3.1], therefore we will give only the part which is essentially different. The modification is based on an estimation of the coefficients $\bar{a}_{k}$ which also leads to the inequality (14) from the proof of Theorem 6.3.1 of $[1]$.

So, since

$$
\bar{a}_{k}=\int_{a}^{b} \bar{f}(t) \varphi_{k}(t) d t
$$

then, by the Hölder inequality,

$$
\bar{a}_{k} \leq\left(\int_{a}^{b}|\bar{f}(t)|^{p} d t\right)^{1 / p}\left(\int_{a}^{b}\left|\varphi_{k}(t)\right|^{p^{\prime}} d t\right)^{1 / p^{\prime}}
$$


and consequently, by our assumption,

$$
\begin{aligned}
1 & =\sum_{k=1}^{r}\left|\bar{a}_{k}\right|^{p^{\prime}} \leq \sum_{k=1}^{r} \int_{a}^{b}\left|\varphi_{k}(t)\right|^{p^{\prime}} d t\left(\int_{a}^{b}|\bar{f}(t)|^{p} d t\right)^{p^{\prime} / p} \\
& \leq(b-a) \sum_{k=1}^{r}\left(\frac{1}{b-a} \int_{a}^{b}\left|\varphi_{k}(t)\right|^{q} d t\right)^{p^{\prime} / q}\left(\int_{a}^{b}|\bar{f}(t)|^{p} d t\right)^{p^{\prime} / p} \\
& \leq M^{p^{\prime}} r(b-a)(\Delta(p))^{p^{\prime} / p} .
\end{aligned}
$$

Hence

$$
\Delta(p) \geq \frac{1}{r^{p-1}(b-a)^{p-1} M^{p}},
$$

which is the above mentioned inequality (14).

This modification completes the proof of $1^{\circ}$.

The proof of $2^{\circ}$ is based on the proof of $1^{\circ}$, so it is exactly the same as that in [1].

3. Remark. This version of the assumption in the theorem of F. Riesz is sometimes more useful in applications, e.g. in investigation of strong summability of orthogonal expansions.

\section{References}

[1] S. Kaczmarz and H. Steinhaus, Theory of Orthogonal Series, Moscow, 1958 (in Russian).

[2] J. Marcinkiewicz and A. Zygmund, Some theorems on orthogonal systems, Fund. Math. 28 (1937), 309-335.

[3] B. Osilenker, Fourier Series in Orthogonal Polynomials, World Sci., Singapore, 2001.

[4] F. Riesz, Über eine Verallgemeinerung der Parsevalschen Formel, Math. Zeit. 18 (1928), $117-1124$. 